\title{
On the Collaborative Governance of Decentralized Edge Microclouds with Blockchain-based Distributed Ledgers
}

\author{
Felix Freitag \\ Department of Computer Architecture. Technical University of Catalunya. Barcelona, Spain \\ \{felix\}@ac.upc.edu
}

\begin{abstract}
Today's commercial model for edge computing services consists in lightweight devices at the network edge connected through the Internet to remote cloud data centers. Microclouds are an alternative vision of edge computing, where the cloud infrastructure runs at the network edge leveraging decentralized resource contributions of a community. But current attempts to build such microclouds lack a collaborative governance system to operate successfully. In this paper we discuss the opportunity to implement with blockchain technologies key services to enable the decentralized collaborative governance of microclouds. A multiagent approach could further contribute to improve the efficiency in the decision making in the collaborative governance service.

Index Terms - edge cloud computing; decentralized clouds;
\end{abstract}

\section{INTRODUCTION}

Community networks are collectively built communication networks. They are constructed bottom-up by a community and maintained by their users. One successful case of such a collaboratively built network is Guifi.net ${ }^{1}$, located around Barcelona in Spain (Figure 1). Guifi.net network started in 2004 and has today more than 30.000 nodes, which makes it the largest community network worldwide [10].

Running collaboratively community microcloud to host services and applications locally in Guifi.net has not yet consolidated. Practical efforts which were undertaken, e.g. developing the Cloudy platform ${ }^{2}$, have not achieved to engage a larger number of participants [3]. Different to the edge microclouds, other volunteer contributed infrastructures in Guifi.net, like the network infrastructure, have been achieved to be sustainable. The presence of commercial ISPs operating within Guifi seems to play an important role for this sustainability at the network level [4].

Blockchain-based distributed ledgers are designed as decentralized systems [12]. By using the computing resources of independent organizations, the service of a trusted and immutable data store is provided. Besides the popular use case of cryptocurrencies, other applications implemented though smart contracts were proposed for blockchain platforms. While for several blockchain-based services a centralized service implementation might as well exist, an advantage of the

\footnotetext{
${ }^{1}$ http://guifi.net/

${ }^{2} \mathrm{http} / / /$ cloudy.community/
}

blockchain-based service can be that is is not centrally controlled.

Multi-agent system (MAS) have been applied in cloud computing to improve the performance of a cloud system [11]. Software agents can pursue the goals of individual actors in the system and they can improve through the interaction with other agents the overall efficiency of the cloud system.

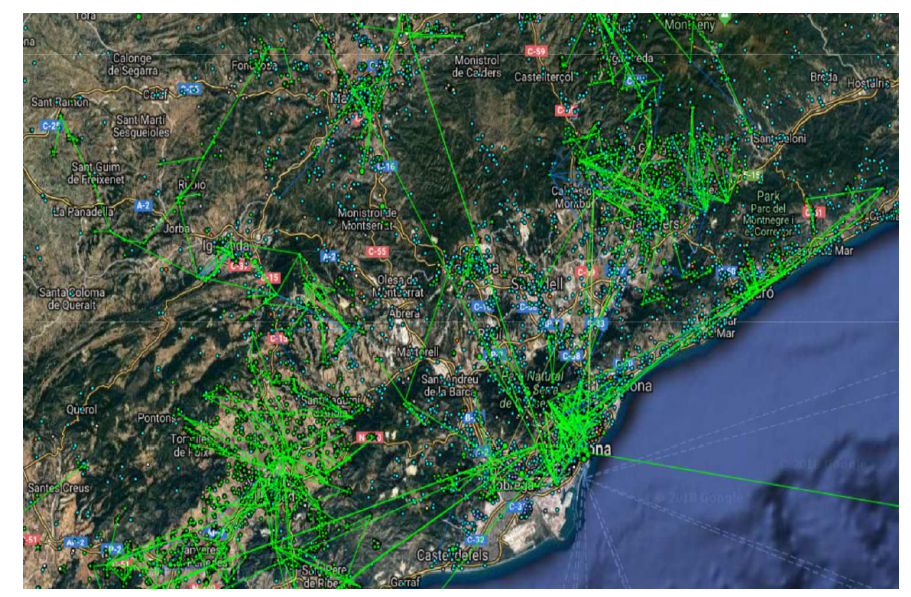

Fig. 1. Guifi.net nodes and links in the area of Barcelona.

In this paper we introduce collaborative governance for microclouds and the opportunity for leveraging blockchain technology to support the decentralized implementation of such a collaborative governance system. In particular we aim to elaborate on:

- The potential of decentralized community microclouds for edge computing to motivate this use case for being supported by a blockchain-based collaborative governance service.

- The challenges and requirements that need to be satisfied by an implementation of the governance system.

- How blockchain-based distributed ledgers could implement specific support services and how a multi-agent system could contribute to the efficiency of the collaborative governance service. 


\section{THE USE CASE OF DECENTRALIZED COMMUNITY MICROCLOUDS}

\section{A. Positioning the approach of edge microclouds}

The collaborative community infrastructure which we target at aims to leverage the contributions of computing resources to build a microcloud at the network edge. In community edge microclouds, the infrastructure consists of a pool of distributed resources on which shared services are run for and by its participants.

P2P systems have been used in the Internet by millions of people for file sharing applications. Different to $\mathrm{P} 2 \mathrm{P}$ applications, community microclouds are not application-specific, e.g. used for file sharing only, but can generally run any distributed application over the shared resource pool. Similar to $\mathrm{P} 2 \mathrm{P}$ applications, community microclouds are decentralized and do not have a centralized governance, every participant can contribute resources and consume services.

Volunteer computing also relies on the collaboration of computing device owners to support a common goal [1]. Similar to volunteer computing, in microclouds running useful services could be a mean to encourage contribution and participation of users. Different to volunteer computing, the services that microclouds could target are those that are required by the community of the users they are to serve.

In today's commerical edge computing model, a lightweight computing device is located close to the user [9]. Commercial edge cloud computing, however, is organized differently to community microclouds. In a typical commercial edge computing scenario, an edge device is a resource that extends the capabilities of a data center-based cloud service.

Blockchain platforms are designed to leverage on decentralized contributions of computing resources. As such they need to gather decentralized resources to build the infrastructure on which the blockchain platform is operated. This concept is similar to that of building collaboratively a decentralized community microcloud, which also requires the contribution of resources of the participants. Differently however, the motivation for the participation in the blockchain platform, e.g. the participation as a miner, is profit-driven and not motivated by any social purpose. The motivation of a service providers that uses a blockchain platform may be motivated by saving costs, e.g. by the reduced cost for running an offered service over the blockchain platform than paying for a centralized service provision. In community microclouds there are currently no monetary rewards and the reason to participate seems to be the support of social goals.

Comparing edge microclouds with the above discussed systems in terms of usage, however, we observe that all these other approaches have achieved to attract a huge number of participants. We suggest collaborative governance services as a mean to increase the users interest and potential of edge microclouds.
B. Specific challenges and requirements for building collaborative governance services for community microclouds

We discuss a list of characteristics which we propose for this collaborative governance system. The identified characteristics are inspired by the elements which already contribute successfully to the sustainability of the Guifi.net network infrastructure [4], and by our practical experience with the development and operation of Cloudy nodes in Guifi.net [3]. The proposed governance system is envisioned to run as an additional service on the microcloud infrastructure itself and needs to adapt to its constraints, and not as an additional external infrastructure.

1) Multi-tenancy of the shared resource: A computing node, which is contributed by a member to the community network, is often used as a shared device [2]: On one hand, the device is used for the benefit of the community, e.g. by doing some monitoring function for the network. On the other hand, the device runs personal services for the node owner. As long as the community service operation is transparent to the node owner, i.e. without noticed service degradation, a collaborative effort will be made. Service degradation, however, may influence on the user's willingness to make the resource contribution.

2) Lightweight computing device: Computing devices run by volunteers in a $24 / 7$ mode at the user's premises need to have a low energy consumption in order to be accepted. Typical computing devices include popular Single-BoardComputers (SBCs) like Raspberry Pi and Atom-based miniComputers $^{3}$. These devices are less performing than typical desktop PCs, and weaker in terms of RAM, computing power and disk space. This fact has to be taken into account for the hardware requirement of the governance service. There is, however, an important body of works which proposes ways to increase the capabilities of low-power devices as edge nodes, e.g. [5].

3) End user friendliness: We must consider the diversity of backgrounds in the members of a community which runs a microcloud. Different levels of technical skills exist and the participation in edge microclouds must be designed to be inclusive, i.e. there must not be entry barriers by requiring skills for complex usage and operation.

Self-management capabilities of the governance services is therefore an important requirement. Another related requirement is that the governance system should be usable by a large number of people. For instance the administrative burden to run a community microcloud node must be reduced. There are several platforms that exemplify easy service deployment targeting at end users ${ }^{4} 5$.

In the following section we propose a collective governance system for community edge microclouds, taking into account the successful elements of the previously discussed systems and the specific challenges.

\footnotetext{
${ }^{3}$ Many of the Cloudy nodes in Guifi.net are Minix mini-PCs. http://cloudy community/2015/09/

${ }^{4}$ Sandstorm. https://sandstorm.io/

${ }^{5}$ YunoHost. https://yunohost.org/\#/
} 


\section{Governance Service Proposal}

\section{A. Architectural design and implementation options}

The architectural design we present builds upon the design of the Cloudy software platform [7] developed by Guifi.net. For this we refine the available support services and aim to extend Cloudy with collaborative governance services. The architecture is organized into the three layers front end, governance services and support services (Figure 2).

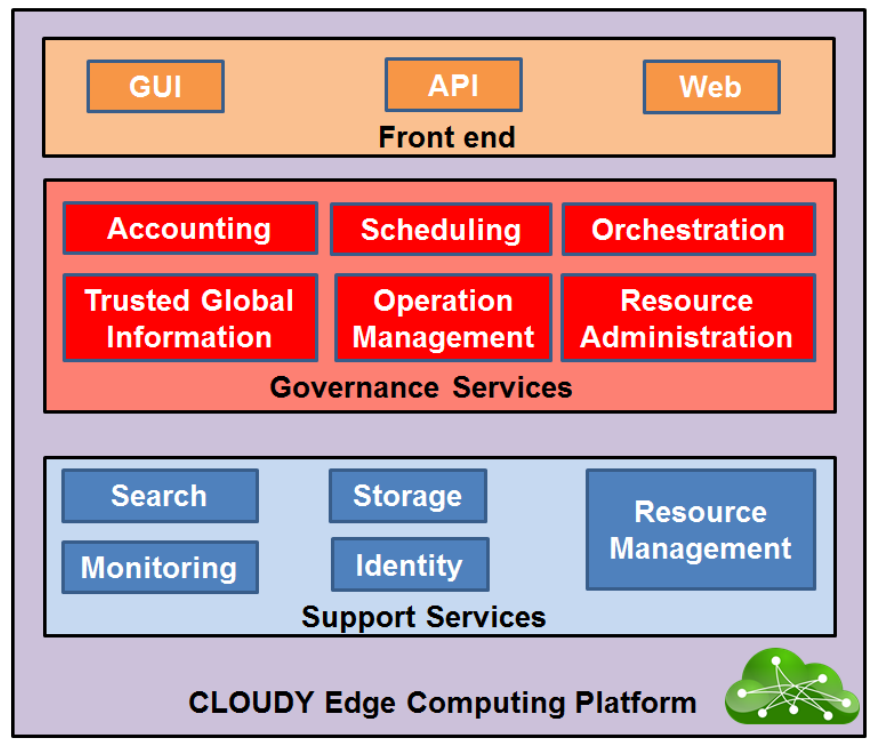

Fig. 2. Technical layers for the collaborative governance of edge microclouds.

The front end layer provide a user-friendly Web interface to perform operations on the community cloud node. The API aims to support automated node-to-node operation. The Web interface available in Cloudy is the main tool for the user to search and deploy services.

The governance service layer contains components for accounting of usage, resources and participation, as well as operations which are fed from the processing of this information. For the implementation, distributed ledgers seem to be a suitable option specifically for the following two components:

Accounting: Service usage and service contributions of community cloud members should be registered by the system in order for the community to be able to provide feedback and potentially rewards. The requirements for this component include that the information provided to take decisions is trusted by all participants. The component receives input from the monitoring component (support service layer). The workload of the current accounting data can be considered as low, since changes in the service offers in the Cloudy community clouds are not very frequent. The computational requirements to perform this trusted accounting on a device should be low in order for the service execution to be transparent for the owner of a node. A distributed ledger offered by a permissioned blockchain platform could be considered as a solution to implement this component.
Trusted Global Information: The overall system status (e.g. computational resource usage, service usage and offer) should be registered in a distributed ledger in order to provide information to the participants. This feedback could serve for the community to take informed decisions. It could also provide information to feed into social network channels to document usage and benefits.

With regards to the support service layer, the components are available in Cloudy, but developments in blockchain technology may require adapting them for better integration. For instance, the identity service could leverage ideas of Sovrin ${ }^{6}$ for being improved. The storage service could be extended by IPFS ${ }^{7}$. An IPFS storage layer combined with a blockchain platform could reduce the cost of smart contract execution on the blockchain.

\section{B. The multi-agent approach to enhance the governance of Cloudy microclouds}

A community microcloud is a decentralized system with multiple owners of individual nodes which donate resources to a common resource pool that forms the microcloud infrastructure. The collaborative governance system aims to implement a set of services to achieve a higher attractiveness of microclouds by improved performance. We can identify several options for contributions by a multi-agent system (MAS):

1) Autonomous agents acting in behalf of the Cloudy providers and users: Currently, the preferences of a Cloudy node owner on the node operation are not delegated to a software agent. The need for multi-tenancy of a Cloudy node, however, is already recognized (see section II-A) and individual profiles to represent the specific preferences of a user could be created, based on which a software agent could interact with other agents. Research on user models and how they are integrated in multi-agent negotiation was done for instance in [8]. It was shown that agents contributed to a higher fulfillment of the user preferences.

2) Multi-agent system to support the cloud: In [11] the potential of agents to improve cloud performance was indicated. In microclouds, more intelligent decisions to determine resource allocations by MAS could be very relevant. Resources in a microcloud are lightweight and heterogeneous. Therefore, appropriate decisions are needed to achieve an efficient and performing system.

3) End-user friendliness: There are several roles in community microclouds. The actors participating in microclouds can take roles which are similar to those of data center clouds. Node owners can act as service providers, e.g. offer an application as SaaS. At the same time, node owners can be consumers of services offered by other nodes. The microcloud infrastructure itself, however, does not have a single owner and consist of those resources and services which are donated to the community [3]. In [6] the understanding of QoE as a

\footnotetext{
${ }^{6}$ Sovrin: Identity For All. https://sovrin.org/

${ }^{7}$ https://ipfs.io/
} 
multiple dimensional construct was presented. This view to manage QoE could also be considered if applicable to the conditions of microclouds.

\section{Cloudy node with services}

The Cloudy platform has been started to operate on edge nodes in Guifi.net in 2015 and there are around 30 operational nodes. Figure 3 shows a Cloudy node deployed on a mini-PC.

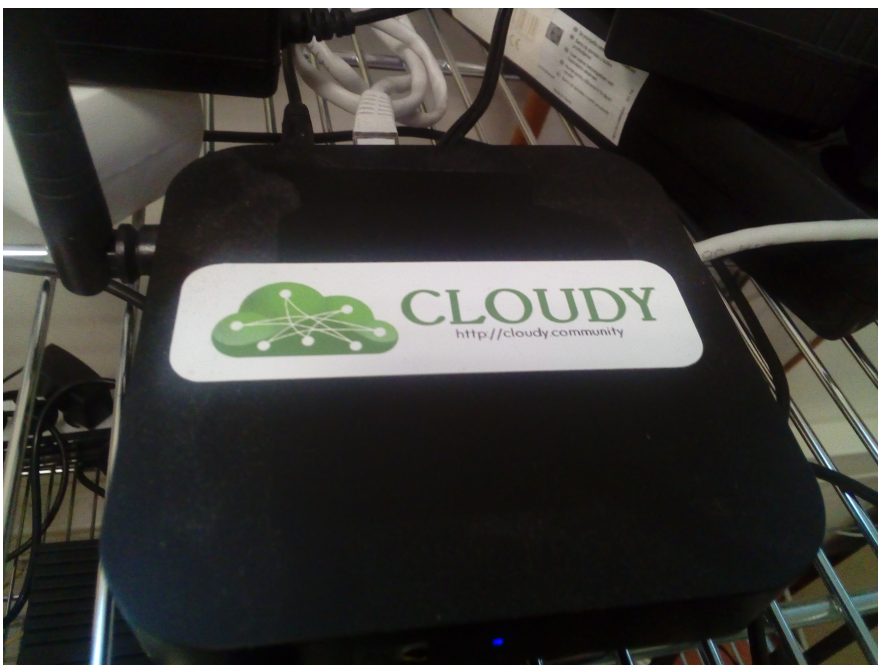

Fig. 3. Cloudy device.

In Figure 4 the Web interface of Cloudy is shown. The sceenshot shows a multichain blockchain container deployed in Cloudy with docker-compose. The proposed components of the governance service are aimed to extend the Cloudy platform.

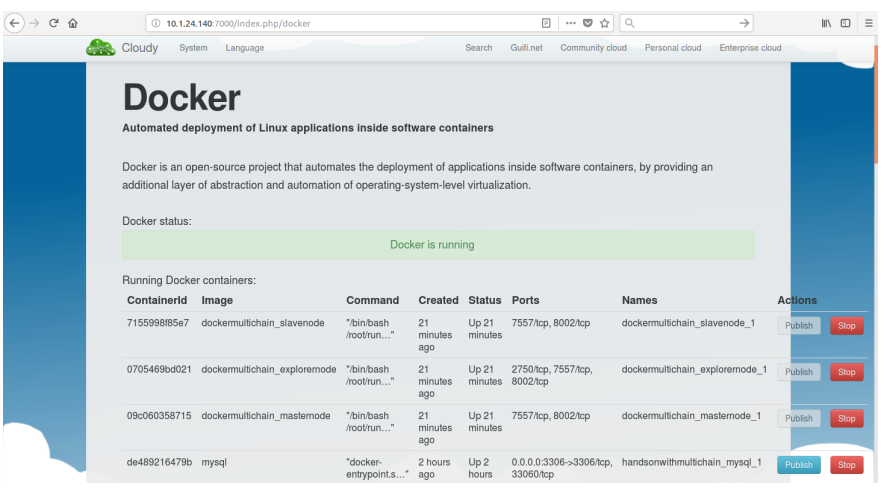

Fig. 4. Multichain node deployed in Cloudy.

\section{CONCLUSION}

We presented the use case of community edge microclouds. They currently lack a collaborative goverance layer. Several services of this governance layer could be implemented by a trusted and immutable distributed ledger, which blockchain technology can offer. As such, blockchain technology is suggested as technical enabler for building the collaborative governance services.
Operating the collaborative governance service for a distributed and decentralized computing infrastructure at the network edge provides specific challenges, such as fulfilling the multi-tenancy purpose of the microcloud nodes, to offer suitable performance on lightweight computing devices, and to be end user friendly. A multi-agent system has the potential to improve the efficiency of the microcloud governance service by determining through interactions among agents more appropriate decisions with regards to individual and global performance goals.

Future work aims to extend the Cloudy platform with a governance service and to explore a MAS approach for efficient usage of the governance service. Economic and legal issues are other areas which need to be addressed to consolidate the design of edge microclouds.

\section{ACKNOWLEDGMENT}

This work was partially supported by the European Unions Horizon 2020 research and innovation programme under the project LightKone (H2020-732505), the project netCommons (H2020-688768), and by the Spanish government under contract TIN2016-77836-C2-2-R.

\section{REFERENCES}

[1] D. P. Anderson, "Volunteer computing: The ultimate cloud," $X R D S$, vol. 16, no. 3, pp. 7-10, Mar. 2010. [Online]. Available: http: //doi.acm.org/10.1145/1734160.1734164

[2] N. Apolnia, F. Freitag, and L. Navarro, "Leveraging deployment models on low-resource devices for cloud services in community networks,' Simulation Modelling Practice and Theory, vol. 77, pp. 390 - 406, 2017. [Online]. Available: http://www.sciencedirect.com/science/article/ pii/S1569190X15303002

[3] R. Baig, F. Freitag, and L. Navarro, "Cloudy in guifi.net: Establishing and sustaining a community cloud as open commons," Future Generation Computer Systems, vol. 87, pp. 868 - 887, 2018. [Online]. Available: http://www.sciencedirect.com/science/article/pii/ S0167739X1732856X

[4] R. Baig, R. Roca, F. Freitag, and L. Navarro, "guifi.net, a crowdsourced network infrastructure held in common," Computer Networks, vol. 90, pp. $150-165,2015$, crowdsourcing. [Online]. Available: http: //www.sciencedirect.com/science/article/pii/S1389128615002327

[5] Y. Elkhatib, B. Porter, H. B. Ribeiro, M. F. Zhani, J. Qadir, and E. Rivire, "On using micro-clouds to deliver the fog," IEEE Internet Computing, vol. 21, no. 2, pp. 8-15, Mar 2017.

[6] E. Kafetzakis, H. Koumaras, M. A. Kourtis, and V. Koumaras, "Qoe4cloud: A qoe-driven multidimensional framework for cloud environments," in 2012 International Conference on Telecommunications and Multimedia (TEMU), July 2012, pp. 77-82.

[7] A. M. Khan and F. Freitag, "On edge cloud service provision with distributed home servers," in 2017 IEEE International Conference on Cloud Computing Technology and Science (CloudCom), Dec 2017, pp. 223-226.

[8] A. Najjar, C. Gravier, X. Serpaggi, and O. Boissier, "Modeling user expectations amp;amp; satisfaction for saas applications using multiagent negotiation," in 2016 IEEE/WIC/ACM International Conference on Web Intelligence (WI), Oct 2016, pp. 399-406.

[9] M. Satyanarayanan, "The emergence of edge computing," Computer, vol. 50, no. 1, pp. 30-39, Jan 2017.

[10] M. Selimi et al., "Cloud services in the guifi.net community network," Computer Networks, vol. 93, Part 2, pp. 373 - 388, 2015, community Networks. [Online]. Available: http://www.sciencedirect.com/science/ article/pii/S1389128615003175

[11] D. Talia, "Clouds meet agents: Toward intelligent cloud services," IEEE Internet Computing, vol. 16, no. 2, pp. 78-81, March 2012.

[12] A. K. White, Blockchain: Discover the Technology behind Smart Contracts, Wallets, Mining and Cryptocurrency. CreateSpace Independent Publishing Platform, 2018. 